\title{
Complexity of dynamical sphere in self-interacting Brans-Dicke gravity
}

\author{
M. Sharif ${ }^{\mathrm{a}}\left(\mathbb{D}\right.$, Amal Majid $^{\mathrm{b}}$ \\ Department of Mathematics, University of the Punjab, Quaid-e-Azam Campus, Lahore 54590, Pakistan
}

Received: 2 November 2020 / Accepted: 9 December 2020 / Published online: 23 December 2020

(C) The Author(s) 2020

\begin{abstract}
This paper aims to derive a definition of complexity for a dynamic spherical system in the background of self-interacting Brans-Dicke gravity. We measure complexity of the structure in terms of inhomogeneous energy density, anisotropic pressure and massive scalar field. For this purpose, we formulate structure scalars by orthogonally splitting the Riemann tensor. We show that self-gravitating models collapsing homologously follow the simplest mode of evolution. Furthermore, we demonstrate the effect of scalar field on the complexity and evolution of non-dissipative as well as dissipative systems. The criteria under which the system deviates from the initial state of zero complexity is also discussed. It is concluded that complexity of the sphere increases in self-interacting Brans-Dicke gravity because the homologous model is not shear-free.
\end{abstract}

\section{Introduction}

Numerous astrophysical experiments (Sloan Digital Sky Survey, Large Synoptic Survey Telescope, Two-degree Field Galaxy Redshift Survey) have proved that the mechanism and evolution of the vast universe massively depend on the large scale structures such as stars and galaxies. Thus, the study of these components is vital to gain a better understanding of the cosmos and its origin. These self-gravitating bodies are intricate cosmic objects whose physical properties may undergo a fundamental change due to a slight fluctuation in the interior. Thus, it is necessary to accommodate all the factors contributing to their complicated nature in a relation termed as complexity factor. Such a factor depicts the inter-relationship of various physical parameters (density, pressure, heat dissipation, etc.) as well as gauges the effects of external or internal perturbations on the matter variables.

\footnotetext{
a e-mail: msharif.math@pu.edu.pk (corresponding author)

be-mail: amalmajid89@gmail.com
}

It can also be used to develop a criterion of stability to compare the complexity of different self-gravitating structures. Researchers have explored the concept of complexity many times but a standard definition has not been established [1-4].

Previous definitions of complexity were proposed based on information (distances and symmetries of atomic arrangement) and entropy (quantification of geometrical attributes) of celestial systems. However, these definitions failed to accurately evaluate the complexity of two simple models: ideal gas and perfect crystal. Since the atoms in ideal gas do not occupy fixed positions, therefore, maximum information is required to completely specify any of its probable states. On the other hand, a perfect crystal requires minimum information for its description due to the symmetrical arrangement of atoms. Despite the differences in their geometrical configurations, both physical systems demonstrate minimum complexity. Lopez-Ruiz et al. [5-8] improved the earlier definitions by encompassing the concept of disequilibrium. The main idea was to detect how various probable states differ from the equiprobable distribution of the physical structure. According to this definition, the two systems (ideal gas and a perfect crystal) have zero complexity. The complexity of neutron stars and white dwarfs has been computed by using energy density in place of probability distribution in the above definition [9-12].

Dense stellar systems have tightly packed particles in their interior. This arrangement restricts the movement of nuclear matter in the radial direction. Consequently, radial pressure is less than the force in the transverse direction leading to anisotropy in pressure. Thus, anisotropy plays a significant role in determining the viability and stability of selfgravitating systems. Since the definition proposed by LopezRuiz et al. incorporates energy density only and lacks the contribution of other state determinants (such as anisotropy) therefore, it cannot be considered as the best criterion of complexity. Recently, Herrera [13] devised a new complexity factor for static sphere in the context of general relativity (GR) 
by assuming that the complexity-free system is isotropic as well as homogeneous. The distinguishing feature of Herrera's technique is the integration of the system's active gravitational mass, inhomogeneous energy density and anisotropic pressure in the definition of complexity. He obtained structure scalars through the orthogonal splitting of Riemann tensor to generate the complexity factor.

Herrera's definition of complexity has also been extended for a non-static radiating sphere by minimizing complexity in the mode of evolution [14]. Herrera and his collaborators [15] formulated three complexity factors for an axially symmetric system and examined a possible relation between symmetry and complexity of the setup. They also employed this notion to establish a hierarchy from the simplest (Minkowski) to more complex (radiating) systems [16]. Sharif and Butt computed the complexity factor based on Herrera's approach for a static cylindrically symmetric self-gravitating system [17]. They also investigated the effect of electromagnetic field on the complexity factor of static spherical [18] as well as cylindrical [19] structures and concluded that complexity increases in the presence of charge. The complexity of a charged non-static spherical system has also been explored [20]. Recently, Herrera et al. determined the conditions under which a quasi-homologous system is complexity free [21].

Hubble's discovery of an expanding cosmos has been reconfirmed through recent cosmological observations (redshift and distance-luminosity relationship of type IA Supernovae [22,23]). Cosmological models in GR that explain the evolution of the universe from its origin to its current phase suffer from some drawbacks (like fine-tuning and cosmic coincidence problems). In order to find an adequate solution, researchers modify GR to incorporate the effects of accelerated expansion. Brans and Dicke [24] modified the EinsteinHilbert action and developed a scalar-tensor theory based on Dirac hypothesis and Mach principle. In Brans-Dicke (BD) theory, the gravitational constant is replaced by a dynamical scalar field $\left(\psi(t)=\frac{1}{G(t)}\right)$ whose effect on matter is gauged through a tunable coupling parameter $\left(\omega_{B D}\right)$. The effect of scalar field reduces corresponding to large values of the coupling parameter.

The inflation of the universe is explained by lower values of $\omega_{B D}$ [25] whereas BD gravity is consistent in the weak-field regime for $\omega_{B D} \geq 40,000$ [26]. In order to establish a standard domain of the parameter, a potential function $(V(\Phi))$ is introduced. This function adjusts the values by assigning a mass to the scalar field $(\Phi)$ which leads to an extension of BD gravity known as self-interacting BD (SBD) theory. Sharif and Manzoor formulated structure scalars to study the evolution of dynamical spheres [27,28] and cylinders [29,30,35-38] in SBD theory. Recently, the complexity of different geometries has also been explored by employing Herrera's definition and it was shown that complexity of the self-gravitating structures increases in the presence of a massive scalar field [31-33]. The concept of complexity has been analyzed in other modified theories as well [34]

In this paper, we derive the complexity factor for a dynamical dissipative sphere by considering its pattern of evolution in the background of SBD theory. The paper is organized as follows. In Sect. 2, the SBD field equations and physical variables representing a non-static sphere are evaluated. Structure scalars are derived from the orthogonal splitting of the Riemann tensor in Sect. 3. Section 4 gives an overview of the complexity and evolution of the system. Kinematical quantities and solutions corresponding to non-dissipative and dissipative fluids are determined in Sect. 5. We discuss stability of the vanishing complexity condition in Sect. 6. In the last section, we summarize the main results.

\section{Self-interacting Brans-Dicke theory and matter variables}

Self-interacting BD theory is defined via the action (with $\left.8 \pi G_{0}=1\right)$

$S=\int \sqrt{-g}\left(\mathcal{R} \Phi-\frac{\omega_{B D}}{\Phi} \nabla^{\mu} \nabla_{\mu} \Phi-V(\Phi)+L_{m}\right) d^{4} x$

where the Ricci scalar, determinant of metric tensor and matter Lagrangian are represented by $\mathcal{R}, g$ and $L_{m}$, respectively. The SBD field and wave equations derived through the variation of above action are, respectively, given as

$$
\begin{aligned}
G_{\mu \nu} & =T_{\mu \nu}^{(\mathrm{eff})}=\frac{1}{\Phi}\left(T_{\mu \nu}^{(m)}+T_{\mu \nu}^{\Phi}\right), \\
\square \Phi & =\frac{T^{(m)}}{3+2 \omega_{B D}}+\frac{1}{3+2 \omega_{B D}}\left(\Phi \frac{d V(\Phi)}{d \Phi}-2 V(\Phi)\right),
\end{aligned}
$$

where the matter distribution is described by the energymomentum tensor $T_{\mu \nu}^{(m)}$ with $T^{(m)}=g_{\mu \nu} T_{\mu \nu}^{(m)}$. The effects of massive scalar field are introduced in the matter source through the following energy-momentum tensor

$$
\begin{aligned}
T_{\mu \nu}^{\Phi}= & \Phi_{, \mu ; \nu}-g_{\mu \nu} \square \Phi+\frac{\omega_{B D}}{\Phi}\left(\Phi_{, \mu} \Phi_{, \nu}-\frac{g_{\mu \nu} \Phi_{, \alpha} \Phi^{, \alpha}}{2}\right) \\
& -\frac{V(\Phi) g_{\mu \nu}}{2}
\end{aligned}
$$

where $\square \Phi=\Phi_{; \mu}^{, \mu}$. We consider a collapsing sphere bounded by a hypersurface $\Sigma$ represented in comoving co-ordinates as

$$
\begin{aligned}
d s^{2}= & -A(r, t)^{2} d t^{2}+B(r, t)^{2} d r^{2} \\
& +R(t, r)^{2}\left(d \theta^{2}+\sin ^{2} \theta d \phi^{2}\right) .
\end{aligned}
$$

The energy density $(\rho)$, radial $\left(p_{r}\right) /$ transverse $\left(p_{\perp}\right)$ pressures and heat flux $\left(q_{\mu}\right)$ of the anisotropic collapsing sphere are 
specified by the following energy-momentum tensor

$$
\begin{aligned}
T_{\mu \nu}^{(m)}= & \left(\rho+p_{\perp}\right) u_{\mu} u_{\nu}+p_{\perp} g_{\mu \nu} \\
& +\left(p_{r}-p_{\perp}\right) s_{\mu} s_{\nu}+q_{\mu} u_{\nu}+u_{\mu} q_{\nu},
\end{aligned}
$$

where the 4-velocity $\left(u_{\mu}=(-A, 0,0,0)\right)$, radial 4-vector $\left(s_{\mu}=(0, B, 0,0)\right)$ and heat flux $\left(q_{\mu}=(0, q B, 0,0)\right)$ obey the following relations

$u^{\mu} u_{\mu}=-1, \quad u^{\mu} q_{\mu}=0, \quad s^{\mu} s_{\mu}=1, \quad s^{\mu} u_{\mu}=0$.

In order to simplify the calculations, we introduce the quantities

$$
\begin{aligned}
\Pi_{\mu \nu} & =\Pi\left(s_{\mu} s_{\nu}-\frac{h_{\mu \nu}}{3}\right), \quad P=\frac{1}{3}\left(p_{r}+2 p_{\perp}\right), \\
\Pi & =p_{r}-p_{\perp}, \quad h_{\mu \nu}=g_{\mu \nu}+u_{\mu} u_{\nu},
\end{aligned}
$$

and rewrite the energy-momentum tensor as

$T_{\mu \nu}^{(m)}=\rho u_{\mu} u_{v}+P h_{\mu \nu}+\Pi_{\mu \nu}+q\left(s_{\mu} u_{v}+u_{\mu} s_{\nu}\right)$.

Using Eqs. (2)-(6), the field equations are obtained as

$$
\begin{aligned}
\frac{1}{\Phi}\left(A^{2} \rho-T_{00}^{\Phi}\right)= & \frac{\dot{R}\left(\frac{2 \dot{B}}{B}+\frac{\dot{R}}{R}\right)}{R} \\
\frac{1}{\Phi}\left(-q A B+T_{01}^{\Phi}\right)= & -\frac{A^{2}\left(\frac{R^{\prime 2}}{R^{2}}-\frac{2 B^{\prime} R^{\prime}}{B R}-\frac{B^{2}}{R^{2}}+\frac{2 R^{\prime \prime}}{R}\right)}{B^{2}}, \frac{2 \dot{B} R^{\prime}}{B R}-\frac{2 \dot{R}^{\prime}}{R}, \\
\frac{1}{\Phi}\left(B^{2} p_{r}+T_{11}^{\Phi}\right)= & -\frac{B^{2}\left(\frac{2 \ddot{R}}{R}-\frac{\dot{R}\left(\frac{2 \dot{A}}{A}-\frac{\dot{R}}{R}\right)}{R}\right)}{A^{2}} \\
& +\frac{R^{\prime}\left(\frac{2 A^{\prime}}{A}+\frac{R^{\prime}}{R}\right)}{R}-\frac{B^{2}}{R^{2}}, \\
\frac{1}{\Phi}\left(R^{2} p_{r}+T_{22}^{\Phi}\right)= & -\frac{R^{2}\left(-\frac{\dot{A}\left(\frac{\dot{B}}{B}+\frac{\dot{R}}{R}\right)}{A}+\frac{\dot{B} \dot{R}}{B R}+\frac{\ddot{B}}{B(t, r)}+\frac{\ddot{R}}{R}\right)}{A^{2}} \\
& +\frac{R^{2}\left(\frac{R^{\prime}\left(\frac{A^{\prime}}{A}-\frac{B^{\prime}}{B}\right)}{R}-\frac{A^{\prime} B^{\prime}}{A B}+\frac{A^{\prime \prime}}{A}+\frac{R^{\prime \prime}}{R}\right)}{B^{2}},
\end{aligned}
$$

where

$$
\begin{aligned}
T_{00}^{\Phi}= & -\dot{\Phi}\left(\frac{2 \dot{A}}{A}+\frac{\dot{B}}{B}+\frac{2 \dot{R}}{R}\right)+\frac{A^{2} \Phi^{\prime}\left(\frac{B^{\prime}}{B}+\frac{2 R^{\prime}}{R}\right)}{B^{2}} \\
& +\frac{\omega_{B D}\left(\frac{A^{2} \Phi^{2}}{B^{2}}+\dot{\Phi}^{2}\right)}{2 \Phi}+\frac{A^{2} \Phi^{\prime \prime}}{B^{2}}+\frac{1}{2} V(\Phi) A^{2}, \\
T_{01}^{\Phi}= & -\frac{A^{\prime} \dot{\Phi}}{A}-\frac{\dot{B} \Phi^{\prime}}{B}+\frac{\omega_{B D}}{\Phi} \dot{\Phi} \Phi^{\prime}+\dot{\Phi}^{\prime}, \\
T_{11}^{\Phi}= & -\Phi^{\prime}\left(\frac{A^{\prime}}{A}+\frac{2 B^{\prime}}{B}+\frac{2 R^{\prime}}{R}\right)+\frac{B^{2} \dot{\Phi}\left(\frac{\dot{A}}{A}+\frac{2 \dot{R}}{R}\right)}{A^{2}}
\end{aligned}
$$

$$
\begin{aligned}
& +\frac{\omega_{B D}\left(\frac{B^{2} \dot{\Phi}^{2}}{A^{2}}+\Phi^{\prime 2}\right)}{2 \Phi}+\frac{B^{2} \ddot{\Phi}}{A^{2}}-\frac{1}{2} V(\Phi) B^{2}, \\
T_{22}^{\Phi}= & -\frac{R^{2} \Phi^{\prime}\left(\frac{A^{\prime}}{A}+\frac{B^{\prime}}{B}+\frac{R^{\prime}}{R}\right)}{B^{2}}+\frac{R^{2} \dot{\Phi}\left(\frac{\dot{A}}{A}+\frac{\dot{B}}{B}+\frac{\dot{R}}{R}\right)}{A^{2}} \\
& -\frac{\omega_{B D} R^{2}\left(\frac{\Phi^{\prime 2}}{B^{2}}-\frac{\dot{\Phi}^{2}}{A^{2}}\right)}{2 \Phi}+\frac{R^{2} \ddot{\Phi}}{A^{2}}-\frac{R^{2} \Phi^{\prime \prime}}{B^{2}}-\frac{1}{2} V(\Phi) R^{2} .
\end{aligned}
$$

Here' and ' denote derivatives with respect to the radial and temporal co-ordinates, respectively. The conservation equations corresponding to the anisotropic matter source are expressed as

$$
\begin{aligned}
& \dot{T}_{0}^{0(\text { eff })}+\left(T_{0}^{0 \text { (eff) }}-T_{1}^{1(\text { eff })}\right) \frac{\dot{B}}{B} \\
& +2\left(T_{0}^{0(\mathrm{eff})}-T_{2}^{2(\mathrm{eff})}\right) \frac{\dot{R}}{R}+\left(T_{0}^{1(\mathrm{eff})}\right)^{\prime} \\
& +\left(T_{0}^{1(\mathrm{eff})}\right)\left(\frac{A^{\prime}}{A}+\frac{B^{\prime}}{B}+2 \frac{R^{\prime}}{R}\right)=0, \\
& \dot{T}_{0}^{1(\mathrm{eff})}+\left(T_{1}^{1(\mathrm{eff})}\right)^{\prime}+T_{0}^{1(\mathrm{eff})}\left(\frac{\dot{A}}{A}+\frac{\dot{B}}{B}+2 \frac{\dot{R}}{R}\right) \\
& -\left(T_{0}^{0(\mathrm{eff})}-T_{1}^{1(\mathrm{eff})}\right) \frac{A^{\prime}}{A}+2\left(T_{1}^{1(\mathrm{eff})}-T_{2}^{2(\mathrm{eff})}\right) \frac{R^{\prime}}{R}=0,
\end{aligned}
$$

whereas the wave equation takes the following form

$$
\begin{aligned}
\square \Phi= & \frac{\Phi^{\prime}\left(\frac{A^{\prime}}{A}-\frac{B^{\prime}}{B}+\frac{2 R^{\prime}}{R)}\right)}{B^{2}}-\frac{\dot{\Phi}\left(\frac{-\dot{A}}{A}+\frac{\dot{B}}{B}+\frac{2 \dot{R}}{R}\right)}{A^{2}} \\
& -\frac{\ddot{\Phi}}{A^{2}}+\frac{\Phi^{\prime \prime}}{B^{2}}=\frac{1}{3+2 \omega_{B D}} \\
& \times\left[-\rho+3 P+\left(\Phi \frac{d V(\Phi)}{d \Phi}-2 V(\Phi)\right)\right] .
\end{aligned}
$$

Kinematical quantities (such as 4-acceleration $\left(a_{\mu}\right)$, expansion scalar $(\Theta)$ and shear tensor $\left.\left(\sigma_{\mu \nu}\right)\right)$ are used to study the motion of cosmic objects. These quantities are defined as

$a_{\mu}=u_{\mu ; \nu} u^{\nu}, \quad \Theta=u_{; \mu}^{\mu}, \quad \sigma_{\mu \nu}=u_{\mu ; \nu}+a_{(\mu} u_{\nu)}-\frac{1}{3} \Theta h_{\mu \nu}$,

which for the considered setup turn out to be

$$
\begin{aligned}
a_{1} & =\frac{A^{\prime}}{A}, \quad a^{2}=a_{\mu} a^{\mu}=\left(\frac{A^{\prime}}{A B}\right)^{2}, \\
\Theta & =\frac{1}{A}\left(\frac{\dot{B}}{B}+2 \frac{\dot{R}}{R}\right), \\
\sigma_{11} & =\frac{2}{3} B^{2} \sigma, \quad \sigma_{22}=-\frac{1}{3} R^{2} \sigma,
\end{aligned}
$$

with $a_{\mu}=a s_{\mu}$ and $\sigma=\sqrt{\frac{3}{2} \sigma^{\mu \nu} \sigma_{\mu \nu}}=\frac{1}{A}\left(\frac{\dot{B}}{B}-\frac{\dot{R}}{R}\right)$.

The boundary $(\Sigma)$ of the fluid distribution divides the spacetime into internal and external regions. In order to avoid a discontinuity at the junction, the Darmois conditions must be fulfilled. For this purpose, we assume that outgoing radiations are massless as depicted in Vaidya spacetime given 
by

$$
\begin{aligned}
d s^{2}= & -\left(1-\frac{2 M(v)}{r}\right) d v^{2} \\
& -2 r d r d v+r^{2}\left(d \theta^{2}+\sin ^{2} \theta d \phi^{2}\right),
\end{aligned}
$$

where $M(v)$ and $v$ are the total mass and retarded time, respectively. The matching of the two spacetimes is smooth and continuous when $(m(t, r))_{\Sigma}=(M(v))_{\Sigma},(q)_{\Sigma}=$ $\left(p_{r}\right)_{\Sigma},\left(\Phi_{-}\right)_{\Sigma}=\left(\Phi_{+}\right)_{\Sigma},\left(\Phi_{-}^{\prime}\right)_{\Sigma}=\left(\Phi_{+}^{\prime}\right)_{\Sigma}$ and $\left(\dot{\Phi}_{-}\right)_{\Sigma}=$ $\left(\dot{\Phi}_{+}\right)_{\Sigma}$ [39]. We use Misner and Sharp [40] formula for calculating mass of the collapsing model as

$m=\frac{R^{3}}{2} \mathcal{R}_{232}^{3}=\frac{R}{2}\left[\left(\frac{\dot{R}}{A}\right)^{2}-\left(\frac{R^{\prime}}{A}\right)^{2}+1\right]$,

where $\mathcal{R}_{232}^{3}$ is a component of Riemann tensor $\mathcal{R}_{\beta \gamma \delta}^{\alpha}$. In order to discuss the dynamics of the self-gravitating system, we introduce the proper time and radial derivatives expressed as

$D_{T}=\frac{1}{A} \frac{\partial}{\partial t}, \quad D_{R}=\frac{1}{R^{\prime}} \frac{\partial}{\partial r}$.

The velocity of the collapsing fluid in terms of aerial radius of the spherical surface within the fluid is defined as $U=$ $D_{T} R<0$. The mass and velocity of the sphere are related as

$$
E \equiv \frac{R^{\prime}}{B}=\left(1+U^{2}-\frac{2 m}{R}\right)^{\frac{1}{2}} .
$$

Taking proper time and radial derivative of mass leads to

$$
\begin{aligned}
& D_{T} m=-\frac{R^{2}}{2}\left(\frac{T_{11}^{(\mathrm{eff})}}{B^{2}} U-\frac{T_{01}^{(\mathrm{eff})}}{A B} E\right), \\
& D_{R} m=-\frac{R^{2}}{2}\left(\frac{T_{00}^{(\mathrm{eff})}}{A^{2}}+\frac{T_{01}^{(\mathrm{eff})}}{A B} \frac{U}{E}\right),
\end{aligned}
$$

which imply

$$
\begin{aligned}
\frac{3 m}{R^{3}}= & -\frac{T_{0}^{0(\mathrm{eff})}}{2}+\frac{1}{2 R^{3}} \int_{0}^{r} R^{\prime} R^{3} \\
& \times\left(D_{R} T_{0}^{0 \text { (eff) }}-\frac{3 T_{01}^{(\mathrm{eff})}}{A B R} \frac{U}{E}\right) d r .
\end{aligned}
$$

Tidal forces play a significant role in the evolution of a celestial system. The Weyl tensor $\left(C_{\alpha \beta \sigma}^{\mu}\right)$ incorporates the effects of these forces and is expressed as

$$
\begin{aligned}
C_{\alpha \beta \sigma}^{\mu}= & \mathcal{R}_{\alpha \beta \sigma}^{\mu}-\frac{\mathcal{R}_{\beta}^{\mu}}{2} g_{\alpha \sigma}+\frac{\mathcal{R}_{\alpha \beta}}{2} \delta_{\sigma}^{\mu} \\
& -\frac{\mathcal{R}_{\alpha \sigma}}{2} \delta_{\beta}^{\mu}+\frac{\mathcal{R}_{\sigma}^{\mu}}{2} g_{\alpha \beta}+\frac{1}{6}\left(\delta_{\beta}^{\mu} g_{\alpha \sigma}+g_{\alpha \beta} \delta_{\sigma}^{\mu}\right),
\end{aligned}
$$

where $\mathcal{R}_{\alpha \beta}$ is the Ricci tensor. The Weyl tensor is generally split into electric $\left(E_{\alpha \beta}\right)$ and magnetic $\left(H_{\alpha \beta}\right)$ parts through the 4-velocity of the observer. The magnetic part vanishes in spherical spacetime whereas the electric part reads

$E_{\mu \nu}=C_{\mu \gamma \nu \delta} u^{\gamma} u^{\delta}=\varepsilon\left(s_{\mu} s_{\nu}+\frac{h_{\mu \nu}}{3}\right)$,

where

$$
\begin{aligned}
\varepsilon= & \frac{1}{2}\left(\frac{\left(\frac{R^{\prime}}{R}-\frac{A^{\prime}}{A}\right)\left(\frac{B^{\prime}}{B}+\frac{R^{\prime}}{R}\right)+\frac{A^{\prime \prime}}{A}-\frac{R^{\prime \prime}}{R}}{B^{2}}\right. \\
& \left.+\frac{\frac{\ddot{R}}{R}-\frac{\ddot{B}}{B}-\left(\frac{\dot{A}}{A}+\frac{\dot{R}}{R}\right)\left(\frac{\dot{R}}{R}-\frac{\dot{B}}{B}\right)}{A^{2}}-\frac{1}{R^{2}}\right) .
\end{aligned}
$$

Moreover, the relation

$$
\begin{aligned}
& {\left[\varepsilon-\frac{1}{2}\left(-T_{0}^{0 \text { (eff) }}-T_{1}^{1 \text { (eff) }}+T_{2}^{2(\text { eff })}\right)\right]} \\
& \quad=\frac{3 \dot{R}}{R}\left[\frac{1}{2}\left(-T_{0}^{0 \text { (eff) }}+T_{2}^{2(\text { eff })}\right)-\varepsilon\right]-\frac{3 R^{\prime}}{2 R} T_{0}^{1(\text { eff) }},
\end{aligned}
$$

demonstrates the influence of scalar field on energy density, pressure and Weyl tensor.

\section{Structure scalars}

We measure the complexity of the system through structure scalars which are acquired from the orthogonal splitting of Riemann tensors. These quantities were first evaluated by Herrera [41]. Following the same technique, we introduce the following tenors

$$
\begin{aligned}
& Y_{\alpha \beta}=\mathcal{R}_{\alpha \delta \beta \gamma} u^{\delta} u^{\gamma}, \\
& Z_{\alpha \beta}={ }^{*} \mathcal{R}_{\alpha \delta \beta \gamma} u^{\delta} u^{\gamma}=\frac{1}{2} \eta_{\alpha \delta \mu \epsilon} \mathcal{R}_{\beta \gamma}^{\mu \epsilon} u^{\delta} u^{\gamma}, \\
& X_{\alpha \beta}={ }^{*} \mathcal{R}_{\alpha \delta \beta \gamma}^{*} u^{\delta} u^{\gamma}=\frac{1}{2} \eta_{\alpha \delta}^{\mu \epsilon} R_{\mu \epsilon \beta \gamma}^{*} u^{\delta} u^{\gamma},
\end{aligned}
$$

where $\mathcal{R}_{\alpha \beta \delta \gamma}^{*}=\frac{1}{2} \eta_{\mu \epsilon \delta \gamma} \mathcal{R}_{\alpha \beta}^{\mu \epsilon}$ and ${ }^{*} \mathcal{R}_{\alpha \beta \delta \gamma}=\frac{1}{2} \eta_{\alpha \beta \mu \epsilon} \mathcal{R}_{\delta \gamma}^{\mu \epsilon}$ are the right and left duals, respectively. Using Eq. (22), the Riemann tensor can be rewritten in the following form

$$
\mathcal{R}_{\beta \gamma}^{\alpha \delta}=C_{\beta \gamma}^{\alpha \delta}+2 T_{[\beta}^{(\mathrm{eff})[\alpha} \delta_{\gamma]}^{\delta]}+T^{(\mathrm{eff})}\left(\frac{1}{3} \delta_{[\beta}^{\alpha} \delta_{\gamma]}^{\delta}-\delta_{[\beta}^{[\alpha} \delta_{\gamma]}^{\delta]}\right),
$$

and is decomposed as

$$
\begin{aligned}
\mathcal{R}_{\beta \gamma}^{\alpha \delta}= & \mathcal{R}_{(I) \beta \gamma}^{\alpha \delta}+\mathcal{R}_{(I I) \beta \gamma}^{\alpha \delta}+\mathcal{R}_{(I I I) \beta \gamma}^{\alpha \delta} \\
& +\mathcal{R}_{(I V) \beta \gamma}^{\alpha \delta}+\mathcal{R}_{(V) \beta \gamma}^{\alpha \delta},
\end{aligned}
$$

where

$$
\begin{aligned}
\mathcal{R}_{(I) \beta \gamma}^{\alpha \delta}= & \frac{2}{\Phi}\left[\rho u^{[\alpha} u_{[\beta} \delta_{\gamma]}^{\delta]}-P h_{[\beta}^{[\alpha} \delta_{\gamma]}^{\delta]}\right. \\
& \left.+(\rho-3 P)\left(\frac{1}{3} \delta_{[\beta}^{\alpha} \delta_{\gamma]}^{\delta}-\delta_{[\beta}^{[\alpha} \delta_{\gamma]}^{\delta]}\right)\right],
\end{aligned}
$$




$$
\begin{aligned}
\mathcal{R}_{(I I) \beta \gamma}^{\alpha \delta}= & \frac{2}{\Phi}\left[\Pi_{[\beta}^{[\alpha} \delta_{\gamma]}^{\delta]}+q\left(u^{[\alpha} s_{[\beta} \delta_{\gamma]}^{\delta]}+s^{[\alpha} u_{[\beta} \delta_{\gamma]}^{\delta]}\right)\right], \\
\mathcal{R}_{(I I) \beta \gamma}^{\alpha \delta}= & 4 u^{[\alpha} u_{[\beta} E_{\gamma]}^{\delta]}-\epsilon_{\mu}^{\alpha \delta} \epsilon_{\beta \gamma \nu} E^{\mu \nu}, \\
\mathcal{R}_{(I V) \beta \gamma}^{\alpha \delta}= & \frac{2}{\Phi}\left[\Phi_{[; \beta}^{[, \alpha} \delta_{\gamma]}^{\delta]}+\frac{\omega_{B D}}{\Phi} \Phi^{[, \alpha} \Phi_{[, \beta} \delta_{\gamma]}^{\delta]}\right. \\
& -\left(\square \Phi+\frac{\omega_{B D}}{2 \Phi} \Phi_{, \mu} \Phi^{, \mu}+\frac{V(\Phi)}{2}\right) \\
& \left.\times \delta_{[\beta}^{[\alpha} \delta_{\gamma]}^{\delta]}\right], \\
\mathcal{R}_{(V) \beta \gamma}^{\alpha \delta}= & \frac{1}{\Phi}\left[\left(-\frac{\omega_{B D}}{\Phi} \Phi_{, \mu} \Phi^{, \mu}-2 V(\Phi)-3 \square \Phi\right)\right. \\
& \left.\times\left(\frac{1}{3} \delta_{[\beta}^{\alpha} \delta_{\gamma]}^{\delta}-\delta_{[\beta}^{[\alpha} \delta_{\gamma]}^{\delta]}\right)\right] .
\end{aligned}
$$

The structure scalars appear in the trace and trace-free parts of the above quantities as

$$
\begin{gathered}
X_{\alpha \beta}=\frac{X_{T}}{3} h_{\alpha \beta}+X_{\langle\alpha \beta\rangle}, \\
Y_{\alpha \beta}=\frac{Y_{T}}{3} h_{\alpha \beta}+Y_{\langle\alpha \beta\rangle},
\end{gathered}
$$

where

$$
\begin{aligned}
& X_{T}=X_{\alpha}^{\alpha}, \quad X_{\langle\alpha \beta\rangle}=h_{\alpha}^{\mu} h_{\beta}^{\nu}\left(X_{\mu \nu}-\frac{X_{\alpha}^{\alpha}}{3} h_{\mu \nu}\right), \\
& Y_{T}=Y_{\alpha}^{\alpha}, \quad Y_{\langle\alpha \beta\rangle}=h_{\alpha}^{\mu} h_{\beta}^{\nu}\left(Y_{\mu \nu}-\frac{Y_{\alpha}^{\alpha}}{3} h_{\mu \nu}\right) .
\end{aligned}
$$

The four structure scalars in the presence of scalar field turn out to be

$$
\begin{aligned}
X_{T}= & X_{T}^{(\mathrm{m})}+X_{T}^{\Phi}=\frac{1}{\Phi}(\rho) \\
& +\frac{1}{2 \Phi}\left(\frac{5}{2} \square \Phi+\Phi_{, \alpha ; \mu} u^{\alpha} u^{\mu}+\frac{\omega_{B D}}{2 \Phi}\left(\Phi_{, \alpha} \Phi^{, \alpha}\right.\right. \\
& \left.+\Phi_{, \mu} \Phi_{, \alpha} u^{\alpha} u^{\mu}+\frac{21}{2} V(\Phi)\right),
\end{aligned}
$$

$$
\begin{aligned}
X_{T F}= & X_{T F}^{(\mathrm{m})}+X_{T F}^{\Phi}=-\frac{1}{\Phi}\left(\frac{\Pi}{2}+\varepsilon \Phi\right) \\
& +\frac{1}{2 \Phi}\left(\square \Phi+\Phi_{, \alpha ; \mu} u^{\alpha} u^{\mu}+\frac{\omega_{B D}}{2 \Phi}\left(\Phi_{, \alpha} \Phi^{, \alpha}\right.\right. \\
& \left.\left.+\Phi_{, \mu} \Phi_{, \alpha} u^{\alpha} u^{\mu}\right)\right), \\
Y_{T}= & Y_{T}^{(\mathrm{m})}+Y_{T}^{\Phi}=\frac{1}{2 \Phi}\left(\rho+3 p_{r}-2 \Pi\right) \\
& -\frac{1}{2 \Phi}\left(\square \Phi+\Phi_{, \gamma ; \alpha} u^{\gamma} u^{\alpha}\right. \\
& \left.+\frac{\omega_{B D}}{\Phi}\left(\Phi_{, \gamma} \Phi_{, \alpha} u^{\gamma} u^{\alpha}\right)+V(\Phi)\right) \\
Y_{T F}= & Y_{T F}^{(\mathrm{m})}+Y_{T F}^{\Phi}=\frac{1}{\Phi}\left(\varepsilon \Phi-\frac{\Pi}{2}\right) \\
& -\frac{1}{2 \Phi}\left(\square \Phi+\frac{\omega_{B D}}{\Phi}(\Phi, \alpha \Phi, \alpha\right. \\
& \left.\left.+\Phi_{, \gamma} \Phi_{, \beta} u^{\gamma} u^{\beta}\right)+\Phi_{, \gamma ; \mu} u^{\gamma} u^{\mu}\right) .
\end{aligned}
$$

The above equations indicate that $X_{T}$ and $Y_{T}$ govern the total energy density and principal stresses of the system, respectively in the presence of the massive scalar field. Moreover, $X_{T F}$ and $Y_{T F}$ together determine the local anisotropy of the fluid. The impact of anisotropy and inhomogeneity on the evolution of the sphere can be measured through $Y_{T F}$ as

$$
\begin{aligned}
Y_{T F}= & T_{2}^{2(\mathrm{eff})}-T_{1}^{1(\mathrm{eff})}+\frac{1}{2 R^{3}} \\
& \times \int_{0}^{r} R^{\prime} R^{3}\left(-D_{R} T_{0}^{0(\mathrm{eff})}+\frac{3 T_{01}^{(\mathrm{eff})}}{A B R} \frac{U}{E}\right) d r \\
& +\left[\frac{\dot{\Phi}}{A^{2}}\left(\frac{2 \dot{A}}{A}+\frac{3 \dot{R}}{R}\right)-\frac{3 \Phi^{\prime} R^{\prime}}{B^{2} R}\right] .
\end{aligned}
$$

\section{Complexity and evolution of the system}

According to the definition devised in [14], the complexity of the fluid distribution depends on the number of physical factors required to adequately describe its structure. Thus, a spherical object with dust fluid in its interior is less complex as compared to the spherical structure consisting of a perfect fluid. In general, the complexity of a cosmic system depends on various physical properties such as anisotropic pressure and inhomogeneous density. In [31], $Y_{T F}$ was chosen as the complexity factor of the static sphere because it incorporated the essential features of the system and determined their effects on Tolman mass (or active gravitational mass). Equation (34) indicates that $Y_{T F}$ contains the contribution of the significant factors which induce complexity in the current setup. Therefore, we proceed by assuming that the scalar $Y_{T F}$ is the best fit for the complexity factor. Moreover, heat dissipation is an additional factor contributing to the complexity of the dynamical setup. Therefore, it is essential to take into account the pattern of evolution of the system to construct a satisfactory complexity factor. Furthermore, 
in order to minimize the complexity, we will consider the anisotropic fluid evolving through the simplest mode of evolution. For this purpose, we identify two patterns of evolution: homologous and homogeneous.

\subsection{The homologous evolution}

The collapse of a celestial body is homologous if the rate at which matter is pulled to the core is the same throughout, i.e., the velocity of the matter falling inward is directly proportional to the radial distance. On the other hand, if density at the center increases rapidly as compared to other regions, then the cosmic object evolves in a non-homologous pattern. In this section, we derive the condition for a homologous collapse. Heat flow can be expressed in terms of shear and expansion scalars through Eqs.(8) and (18) as

$\frac{1}{2 E \Phi}\left(q-\frac{T_{01}^{\Phi}}{A B}\right)=\frac{1}{3} D_{R}(\Theta-\sigma)-\frac{\sigma}{R}$,

which yields

$D_{R}\left(\frac{U}{R}\right)=\frac{1}{2 E \Phi}\left(q-\frac{T_{01}^{(\mathrm{eff})}}{A B}\right)+\frac{\sigma}{R}$.

Integration of the above equation leads to

$U=R \int_{0}^{r} R^{\prime}\left[\frac{1}{2 E \Phi}\left(q-\frac{T_{01}^{(\mathrm{eff})}}{A B}\right)+\frac{\sigma}{R}\right] d r+c(t) R$,

where $c(t)=\frac{U_{\Sigma}}{R_{\Sigma}}$ is an integration function. If the fluid is nondissipative and shear-free then the integral in the above equation vanishes providing the necessary condition of homologous evolution $U \sim R$ [42-44]. Thus, the ratio of aerial radii of any two concentric circles must be constant. It is evident from the homologous condition that $R$ is a separable function of $t$ and $r$. The homologous condition corresponding to the current setup is

$\frac{1}{2 E \Phi}\left(q-\frac{T_{01}^{\Phi}}{A B}\right)+\frac{\sigma}{R}=0$.

\subsection{The homogeneous expansion}

The evolution of a cosmic structure is homogeneous if the rate of expansion or collapse is independent of $r$. In other words, homogeneous expansion corresponds to $\Theta^{\prime}=0$. Applying this constraint along with Eqs. (38)-(35) implies

$D_{R} \sigma=0$,

which leads to $\sigma=0$ (due to the regularity conditions at the core). Thus, Eq. (35) yields

$q=\frac{T_{01}^{\Phi}}{A B}$, i.e., the fluid is dissipative. It must be noted that in GR, a shear-free matter distribution evolving under the condition $\Theta^{\prime}=0$ must also be non-dissipative and consequently, homologous.

\section{Kinematical variables}

In this section, we analyze the behavior of different physical quantities to choose the simplest pattern of evolution. Imposing the homologous condition on Eq. (35) produces

$(\Theta-\sigma)^{\prime}=\left(\frac{3 \dot{R}}{A R}\right)^{\prime}=0 \Rightarrow A^{\prime}=0$.

Thus, the homologous fluid is geodesic $(a=0)$ in the current scenario. This implies that homologous pattern can be considered as the simplest mode of evolution. Without loss of generality, we take $A=1$. Conversely, the geodesic condition produces

$(\Theta-\sigma)=\frac{3 \dot{R}}{R}$.

Successive derivatives with respect to $r$ close to the center imply that the fluid is homologous [14].

It must be noted that the counterpart of this structure in GR is shear-free when $q=0$. However, in the presence of scalar field, the non-dissipative as well as homologous fluid is geodesic but not shear-free as

$\sigma=\frac{R T_{01}^{\Phi}}{2 R^{\prime}}$

If the non-dissipative fluid undergoes homogeneous expansion, then Eq. (39) implies $T_{01}^{\Phi}=0$. Moreover, shear scalar is evaluated from Eq. (35) as

$\sigma=\frac{3}{2 R^{3}} \int_{0}^{r} \frac{R^{3}}{A} T_{01}^{\Phi} d r+\frac{g(t)}{R^{3}}=\frac{g(t)}{R^{3}}$,

where $g(t)$ is an arbitrary function of integration. Since at the center $R=0$ therefore, $g(t)$ must be zero. It follows that in the non-dissipative case, homogeneous expansion implies homologous evolution (since $T_{01}^{\Phi}=0 \Rightarrow \sigma=0 \Rightarrow U \sim R$ ). Conversely, if $\sigma=\frac{R T_{01}^{\Phi}}{2 R^{\prime}}$ then $\Theta^{\prime}=\left(\frac{R T_{01}^{\Phi}}{2 R^{\prime}}\right)^{\prime}$. Thus, homologous evolution implies homogeneous expansion only if $T_{01}^{\Phi}=0$. In the subsequent sections, we obtain solutions satisfying the conditions for vanishing complexity as well as homologous fluid. For this purpose, we assume an exponential form of the scalar field as $\Phi(t, r)=\Phi(t)=\Phi_{0} t^{b}$, where $b$ is a constant and $\Phi_{0}$ is the present day value of the scalar field. 


\subsection{Case $1: q=0$}

We first consider the non-dissipative case. It is worthwhile to mention here that the homologous fluid for the chosen scalar field satisfies $T_{01}^{\Phi}=0$. Hence, in the non-dissipative case, there is a unique criterion for the simplest evolution (since homologous evolution fulfils the conditions of homogeneous expansion and vice versa). The homologous condition yields

$$
B(t, r)=g_{1}(r) R(t, r),
$$

where $g_{1}(r)$ is an arbitrary function of integration. Employing the above relation in the condition of vanishing complexity and wave equation generates the following expressions

$$
\begin{aligned}
V(\Phi)= & \frac{\Phi_{0} t^{\beta-2}}{h(r)^{3} R^{4}}\left[2 t^{2} R^{\prime}\left(h(r) R^{\prime}+h^{\prime}(r)\right)\right. \\
& -2 t^{2} h(r) R R^{\prime \prime}+h(r)^{3} R^{3}(t(5 \beta \dot{R}+4 t \ddot{R}) \\
& \left.\left.+\beta\left(\beta\left(\omega_{B D}+2\right)-2\right) R\right)+2 t h(r)^{3} R^{2} \dot{R}(t \dot{R}+\beta R)\right], \\
& \frac{\Phi_{0} t^{\beta-1}}{\beta\left(2 \omega_{B D}+3\right) h(r) R}\left[2 t ^ { 2 } h ^ { \prime } ( r ) \left(-2 \beta R^{\prime} R^{2}\right.\right. \\
& \left.+R\left(R^{\prime}(t \dot{R}+\beta)+t \dot{R}^{\prime}\right)-4 t R^{\prime} \dot{R}\right)+2 t^{2} h(r) \\
& \times\left(R^{2}\left(\beta R^{\prime \prime}-t \dot{R}^{\prime \prime}\right)+t\left(3 R^{\prime \prime} \dot{R}+2 R^{\prime} \dot{R}^{\prime}\right) R\right. \\
& \left.-4 t R^{\prime 2} \dot{R}\right)+h(r)^{3} R^{2}\left(-4 t^{3} \dot{R}^{3}-\beta t^{2}\left(11 \dot{R}^{2}+2\right) R\right. \\
& +t R^{2}\left(\beta\left(6 \beta \omega_{B D}+7 \beta-7\right) \dot{R}+t(5 \beta \ddot{R}+4 t \dddot{R})\right) \\
& \left.\left.+2(\beta-2) \beta\left(\beta \omega_{B D}+\beta-1\right) R^{3}\right)\right]=0 .
\end{aligned}
$$

A complete solution can be determined for a suitable choice of $g_{1}(r)$.

\subsection{Case 2: $q \neq 0$}

In the non-dissipative case, the homologous, zero complexity and wave equations, respectively, read

$$
\begin{aligned}
B= & g_{2}(r) \exp \left(\int_{1}^{t} \frac{\Phi_{0} t^{\beta} R \dot{R}^{\prime}-R^{\prime} \dot{R}}{\left(\Phi_{0} t^{\beta}-1\right) R R^{\prime}} d t\right), \\
V(\Phi)= & \frac{\Phi_{0} t^{\beta-2}}{B^{3} R}\left[2 t^{2} B^{\prime} R^{\prime}+2 t \dot{B} B^{2}(t \dot{R}+\beta R)\right. \\
& -2 t^{2} B R^{\prime \prime}+B^{3}(t(5 \beta \dot{R} 4 t \ddot{R}) \\
& \left.\left.+\beta\left(\beta\left(\omega_{B D}+2\right)-2\right) R\right)\right], \\
& \times \frac{\Phi_{0} t^{\beta-1}}{\beta\left(2 \omega_{B D}+3\right) B R}\left[-6 t^{3} B^{\prime} \dot{B} R R^{\prime}+2 t B^{3}\right. \\
& \times\left(-t^{2} \dot{B} \dot{R}^{2}+t R(\dot{B}(t \ddot{R}-\beta \dot{R})\right. \\
& \left.+t \ddot{B} \dot{R})+\beta\left(\beta \omega_{B D}+\beta-1\right) \dot{B} R^{2}\right) \\
& -2 t^{2} B^{2}\left(R\left(t \dot{B}^{2} \dot{R}-\beta R^{\prime \prime}+t \dot{R}^{\prime \prime}\right)\right. \\
& \left.+\beta \dot{B}^{2} R^{2}-\beta R^{\prime 2}-t R^{\prime \prime} \dot{R}\right) \\
& +2 t^{2} B\left(R \left(B^{\prime}\left(t \dot{R}^{\prime}-\beta R^{\prime}\right)+t\left(2 \dot{B} R^{\prime \prime}\right.\right.\right.
\end{aligned}
$$

$$
\begin{aligned}
& \left.\left.\left.+\dot{B}^{\prime} R^{\prime}\right)\right)-t B^{\prime} R^{\prime} \dot{R}\right)+B^{4} \\
& \times\left(t^{2}\left(-\left(7 \beta \dot{R}^{2}+4 t \ddot{R} \dot{R}+2 \beta\right)\right)+t R(\beta\right. \\
& \left.\times\left(4 \beta \omega_{B D}+5 \beta-5\right) \dot{R}+t(5 \beta \ddot{R}+4 t \dddot{R})\right) \\
& \left.\left.+2(\beta-2) \beta\left(\beta \omega_{B D}+\beta-1\right) R^{2}\right)\right]=0,
\end{aligned}
$$

where $g_{2}(r)$ is an integration function. The above system of equations provide a solution corresponding to an appropriate form of $g_{2}(r)$ for $\Phi(t, r)=\Phi(t)=\Phi_{0} t^{b}$.

\section{Stability of $Y_{T F}=0$ condition}

In this section, we examine whether the state of zero complexity can prevail throughout the evolution of homologous matter distribution for $\Phi(t, r)=\Phi(t)=\Phi_{0} t^{b}$. The evolution of the complexity factor is obtained through Eqs. (11) and (25) as

$$
\begin{aligned}
\dot{Y}_{T F} & +\frac{\dot{\Pi}}{\Phi}+\frac{3 \dot{R}}{R} Y_{T F}+\left(\rho+P_{r}\right) \frac{\sigma}{2 \Phi} \\
& +\frac{1}{2 B \Phi}\left(q^{\prime}-\frac{q R^{\prime}}{R}\right)+\frac{2 \Pi \dot{R}}{R \Phi}+S_{1}=0,
\end{aligned}
$$

where the term $S_{1}$, containing the effects of scalar field, is given as

$$
\begin{aligned}
S_{1}= & \frac{\left(T_{1}^{1 \Phi}-T_{2}^{2 \Phi}\right)}{2 \Phi}-\frac{\left(T_{0}^{1 \Phi}\right)^{\prime}}{\Phi} \\
& -\frac{\left(T_{0}^{1 \Phi}\right)^{\prime}}{2 \Phi}\left(\frac{B^{\prime}}{B}-\frac{R^{\prime}}{R}\right)-\frac{\left(T_{0}^{0 \Phi}-T_{1}^{1 \Phi}\right) \dot{B}}{2 B \Phi} \\
& -\frac{5\left(T_{0}^{0 \Phi}-T_{2}^{2 \Phi}\right) \dot{R}}{2 R \Phi} \\
& -\dot{Y}_{T F}^{\Phi}-Y_{T F} .
\end{aligned}
$$

In the non-dissipative scenario, we assume that $q=\Pi=$ $\sigma=Y_{T F}=0$ at $t=0$ which leads to the following forms of Eq. (41) and its derivative with respect to $t$

$$
S_{1}=-\left(\dot{Y}_{T F}+\dot{\Pi}\right)
$$

$Y_{T F}^{.}+\frac{\ddot{\Pi}}{\Phi}-\frac{\dot{\Pi} \dot{\Phi}}{\Phi^{2}}=3 S_{1}-\dot{S}_{1}+\frac{\dot{\Pi} \dot{R}}{R \Phi}$.

Employing the above relations, the first and second $t$ derivatives of Eq. (34) can be written as

$$
\begin{aligned}
& S_{1}+3\left(\frac{\dot{\Phi} \dot{R}}{R}\right)=\frac{\partial}{\partial t}\left(\int_{0}^{r} R^{3}\left(T_{0}^{0(\mathrm{eff})}\right)^{\prime} d r\right), \\
& 3 S_{1}-\dot{S}_{1}+\frac{\dot{\Pi} \dot{R}}{R \Phi}-3\left(\frac{\dot{\Phi} \dot{R}}{R}\right)^{\cdot \cdot}=\frac{\partial^{2}}{\partial t^{2}}\left(\int_{0}^{r}-R^{3}\left(T_{0}^{0(\mathrm{eff})}\right)^{\prime} d r\right) .
\end{aligned}
$$

We can proceed in the same manner and calculate the higher derivatives of Eq. (34). It is noted that the stability of vanishing complexity depends on state determinants (pressure and energy density) as well as the massive scalar field. Thus, 
anisotropy and inhomogeneity in pressure and energy density, respectively induce complexity in the system. For the general case, i.e., when $q \neq 0$, it can be clearly deduced from Eq. (41) that heat dissipation is an additional factor influencing the $Y_{T F}=0$ condition.

\section{Summary}

Many researchers have explored the dynamics and structure of self-gravitating objects to gain insight into the mechanism of the cosmos. However, the interdependence of physical features (such as energy density, pressure, luminosity, etc.) as well as continuous evolution of astrophysical objects lead to a complicated yet intriguing system. The purpose of this work is to formulate a definition of complexity for non-static systems in the framework of SBD theory. We have considered an anisotropic radiating sphere with inhomogeneous energy density. In order to determine the complexity of the celestial system, we have employed Bel's technique to split Riemann tensor. The resulting elements have yielded scalars that govern the structure of the self-gravitating system. In order to incorporate the dynamical aspect of the non-static regime, we have considered two possibilities for the simplest pattern of evolution: homologous and homogeneous modes. Finally, we have applied the condition of vanishing complexity on homologous distribution to formulate possible solutions for dissipative as well as non-dissipative models. The factors due to which the system can depart from zero complexity during the process of evolution have also been discussed.

The structure scalars evaluated in SBD theory include the massive scalar field and potential function which imply that the scalar field contributes to the complexity of the system. Thus, the SBD spherical system is more complicated than its GR counterpart. The structure scalar $Y_{T F}$ has been selected as an appropriate choice for complexity factor based on the following reasons.

- It has already served as an adequate measure of complexity in the static case [31], thereby ensuring that the current definition of complexity is recovered in the static regime.

- It includes the effects of anisotropy, inhomogeneous energy density and dissipation.

Since the homologous condition has implied that the fluid is geodesic (for both $q=0$ and $q \neq 0$ ) therefore, a homologous pattern of evolution has been chosen to minimize the complexity in the evolution of the system. It is interesting to mention here that the homologous condition includes the effects of the scalar field. Thus, in the non-dissipative case, the complexity factor and shear-tensor do not vanish in contrast to the GR analog [14]. The use of homologous and vanishing complexity conditions for dissipative as well as non-dissipative models have provided open systems that can be closed by choosing suitable integration functions. Furthermore, we have deduced that in SBD gravity the stability of vanishing complexity condition depends on the scalar field in addition to the matter variables (pressure, heat flux, energy density). It is noteworthy to mention here that all the results are recovered for GR [14] under the conditions $\Phi=$ constant and $\omega_{B D} \rightarrow \infty$.

Data Availability Statement This manuscript has no associated data or the data will not be deposited. [Authors' comment: We have not used any observational data in this paper. Consequently, no data will be deposited.]

Open Access This article is licensed under a Creative Commons Attribution 4.0 International License, which permits use, sharing, adaptation, distribution and reproduction in any medium or format, as long as you give appropriate credit to the original author(s) and the source, provide a link to the Creative Commons licence, and indicate if changes were made. The images or other third party material in this article are included in the article's Creative Commons licence, unless indicated otherwise in a credit line to the material. If material is not included in the article's Creative Commons licence and your intended use is not permitted by statutory regulation or exceeds the permitted use, you will need to obtain permission directly from the copyright holder. To view a copy of this licence, visit http://creativecomm ons.org/licenses/by/4.0/.

Funded by SCOAP ${ }^{3}$.

\section{References}

1. A.N. Kolmogorov, Prob. Inf. Theory J. 1, 3 (1965)

2. J. Grassberger, Int. J. Theor. Phys. 125, 907 (1986)

3. P.W. Anderson, Phys. Today 7, 9 (1991)

4. G. Parisi, Phys. World 6, 42 (1993)

5. R. Lopez-Ruiz, H.L. Mancini, X. Calbet, Phys. Lett. A 209, 321 (1995)

6. X. Calbet, R. Lopez-Ruiz, Phys. Rev. E 63, 066116 (2001)

7. R.G. Catalan, J. Garay, R. Lopez-Ruiz, Phys. Rev. E 66, 011102 (2002)

8. J. Sañudo, R. Lopez-Ruiz, Phys. Lett. A 372, 5283 (2008)

9. J. Sañudo, A.F. Pacheco, Phys. Lett. A 373, 807 (2009)

10. KCh. Chatzisavvas et al., Phys. Lett. A 373, 3901 (2009)

11. R.A. de Souza, M.G.B. de Avellar, J.E. Horvath, arXiv:1308.3519

12. M.G.B. de Avellar et al., Phys. Lett. A 378, 3481 (2014)

13. L. Herrera, Phys. Rev. D 97, 044010 (2018)

14. L. Herrera, A. Di Prisco, J. Ospino, Phys. Rev. D 98, 104059 (2018)

15. L. Herrera, A. Di Prisco, J. Ospino, Phys. Rev. D 99, 044049 (2019)

16. L. Herrera, A. Di Prisco, J. Carot, Phys. Rev. D 99, 124028 (2019)

17. M. Sharif, I.I. Butt, Eur. Phys. J. C 78, 850 (2018)

18. M. Sharif, I.I. Butt, Eur. Phys. J. C 78, 688 (2018)

19. M. Sharif, I.I. Butt, Chin. J. Phys. 61, 238 (2019)

20. M. Sharif, S. Tariq, Mod. Phys. Lett. A 35, 28 (2020)

21. L. Herrera, A. Di Prisco, J. Ospino, Eur. Phys. J. C 80, 631 (2020)

22. S. Perlmutter et al., Nature 391, 51 (1998)

23. A.G. Riess et al., Astron. J. 116, 1009 (1998)

24. C. Brans, R.H. Dicke, Phys. Rev. 124, 3 (1961)

25. E.J. Weinberg, Phys. Rev. D 40, 3950 (1989)

26. C.M. Will, Living Rev. Relativ. 4, 4 (2001)

27. M. Sharif, R. Manzoor, Gen. Relativ. Gravit. 47, 98 (2015) 
28. M. Sharif, R. Manzoor, Phys. Rev. D 91, 024018 (2015)

29. M. Sharif, R. Manzoor, Astrophys. Space Sci. 359, 17 (2015)

30. M. Sharif, R. Manzoor, Commun. Theor. Phys. 68, 39 (2017)

31. M. Sharif, A. Majid, Chin. J. Phys. 61, 38 (2019)

32. M. Sharif, A. Majid, Indian J. Phys. (2020)

33. M. Sharif, A. Majid, Int. J. Geom. Methods Mod. Phys. 16, 1950174 (2019)

34. G. Abbas, H. Nazar, Eur. Phys. J. C 78, 510 (2018). ibid. 957

35. G. Abbas, H. Nazar, Astrophys. Space Sci. 364, 11 (2019)

36. M. Sharif, A. Majid, M.M.M. Nasir, Int. J. Mod. Phys. A 34, 32 (2019)
37. M. Zubair, H. Azmat, Int. J. Mod. Phys. D 29, 2 (2020)

38. Z. Yousaf, M.Z. Bhatti, T. Naseer, Phys. Dark Universe 28, 100535 (2020)

39. G. Darmois, Mém. Sci. Math. 25, 58 (1927)

40. C.W. Misner, D.H. Sharp, Phys. Rev. 136, B571 (1964)

41. L. Herrera et al., Phys. Rev. D 79, 064025 (2009)

42. M. Schwarzschild, Structure and Evolution of the Stars (Dover, New York, 1958)

43. R. Kippenhahn, A. Weigert, Stellar Structure and Evolution (Springer, New York, 1990)

44. C. Hansen, S. Kawaler, Stellar Interiors: Physical Principles, Structure and Evolution (Springer, New York, 1994) 